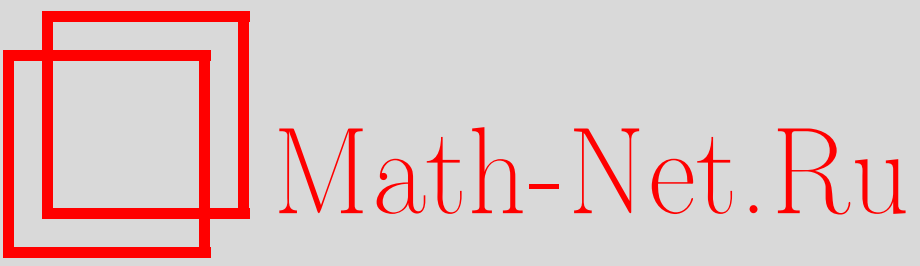

С. Ю. Фаткина, О представлении натурального числа суммой трех почти равных слагаемых, порожденных простыми числами, УМH, 2000, том 55, выпуск 1, 197-198

DOI: https://doi.org/10.4213/rm254

Использование Общероссийского математического портала Math-Net.Ru подразумевает, что вы прочитали и согласны с пользовательским соглашением

http: //www . mathnet.ru/rus/agreement

Параметры загрузки:

IP : 54.224 .135 .184

26 апреля 2023 г., 03:33:10 


\title{
О ПРЕДСТАВЛЕНИИ НАТУРАЛЬНОГО ЧИСЛА СУММОЙ ТРЕХ ПОЧТИ РАВНЫХ СЛАГАЕМЫХ, ПОРОЖДЕННЫХ ПРОСТЫМИ ЧИСЛАМИ
}

\author{
С. Ю. ФАТКИнА
}

В данной статье мы рассмотрим видоизмененную проблему Гольдбаха, а именно тернарную задачу для почти равных слагаемых, принадлежаших различным множествам. Основной результат сформулирован в теореме. Доказательство использует методы работы Г. И. Архипова, К. Буриева, В.Н. Чубарикова [1], а также оценки тригонометрических сумм с простыми числами в коротких интервалах из работы [2].

Теорема. Пусть $N$ - достаточно больиое натуральное число. Тогда для $U=N^{5 / 8} \log ^{c} N$, где с-некоторая константа, диофантово уравнение

$$
\begin{gathered}
N=p_{1}+p_{2}+\left[\sqrt{2} p_{3}\right], \\
\frac{N}{3}-U<p_{i}<\frac{N}{3}+U, \quad i=1,2, \quad \frac{N}{3}-U<\left[\sqrt{2} p_{3}\right]<\frac{N}{3}+U,
\end{gathered}
$$

разрешимо в простых числах $p_{1}, p_{2}, p_{3}$. Более того, число решений этого уравнения представляется асимптотической формулой

$$
T(N, U)=\frac{3 \sqrt{2}}{2} \frac{U^{2}}{\log ^{3} N}+O\left(\frac{U^{2}}{\log ^{4} N}\right) .
$$

ДокАЗАТЕльСТво. Число решений запишем аналитической формулой

$T(N, U)=\int_{0}^{1} S_{1}^{2}(\alpha) S_{2}(\alpha) e^{-2 \pi i \alpha N} d \alpha=\int_{-\Delta}^{\Delta}+\int_{\Delta}^{1-\Delta}=I_{1}+I_{2}, \Delta=N^{\varepsilon} / U(0<\varepsilon<1 / 40)$,

где

$$
S_{1}(\alpha)=\sum_{\frac{N}{3}-U<p<\frac{N}{3}+U} e^{2 \pi i \alpha p}, \quad S_{2}(\alpha)=\sum_{\frac{N}{3}-U<[\sqrt{2} p]<\frac{N}{3}+U} e^{2 \pi i \alpha[\sqrt{2} p]} .
$$

Сначала оценим сверху $I_{2}$. Преобразуем $S_{2}(\alpha)$, воспользовавшись разложением в ряд Фурье $e^{-2 \pi i \alpha\{x\}}$ (см. [3; с. 21, лемма 1]):

$$
S_{2}(\alpha)=\frac{1-e^{-2 \pi i \alpha}}{2 \pi i \log N} \sum_{|m|<M} \frac{1}{\alpha+m} \sum_{\frac{N}{3}-U<[\sqrt{2} p]<\frac{N}{3}+U} \Lambda(n) e^{2 \pi i(\alpha+m) \sqrt{2} p}+O\left(\frac{U}{\sqrt{M}}\right)
$$

$M=N^{1 / 8}$. При определенных $\alpha$ существуют оценки для сумм вида $\sum_{N-A<n<N} \Lambda(n) e^{2 \pi i \alpha n}$.

Лемма 1. Пусть $\alpha=\frac{a}{q}+\frac{\theta}{q \tau}, q \leqslant \tau,|\theta|<1$, и пусть дано любое $B>0$. Тогда существуют $c_{i}>0, i=1,2,3$, такие, что при условиях

$$
\tau=A^{2} N^{-1} \log ^{c_{1}} N, \quad q \geqslant \log ^{c_{2}} N \quad u \quad N^{\frac{3}{5}} \log ^{c_{3}} N \leqslant A \leqslant N
$$

справедлива оченка $\sum_{N-A<n<N} \Lambda(n) e^{2 \pi i \alpha n} \ll A \log ^{-B} N$.

ДокАЗАТЕЛЬСтво см. [2; с. 260, теорема 2]. 
ЛЕмма 2. Пусть $\alpha=\frac{a}{q}+\frac{\theta}{q \tau}, q \leqslant \tau,|\theta|<1$, и пусть даны любие $B>0, c_{i}>0$, $i=1,2$. Тогда существует $c_{3}>0$ такое, что при условиях

$$
\tau=A^{2} N^{-1} \log ^{c_{1}} N, \quad q \leqslant \log ^{c_{2}} N, \quad \frac{|\theta|}{q \tau} \geqslant \frac{\log ^{B+1} N}{A} \quad u \quad N^{\frac{5}{8}} \log ^{c_{3}} N \leqslant A \leqslant N
$$

справедлива оценка $\sum_{N-A<n<N} \Lambda(n) e^{2 \pi i \alpha n} \ll A \log ^{-B} N$.

ДокаЗАТЕЛЬСТво см. [2; с. 260, теорема 3].

При $\alpha$, принадлежащем интервалу $(1 / \tau, 1-1 / \tau)$, либо представление $\alpha$ в виде $\alpha=\frac{a}{q}+\frac{\theta}{q \tau}$, либо представления чисел $\sqrt{2}(\alpha+m)=\frac{a_{m}}{q_{m}}+\frac{\theta_{m}}{q_{m} \tau}(|m|<M)$ удовлетворяют требованиям леммы 1. Значения $\alpha$, принадлежащие интервалам $(\Delta, 1 / \tau)$ и $(1-1 / \tau, 1-\Delta)$, удовлетворяют условиям леммы 2. Следовательно, $F(\alpha)=\min \left(\left|S_{1}(\alpha)\right|,\left|S_{2}(\alpha)\right|\right) \ll U / \log ^{B+1} N$ на интервале $(\Delta, 1-\Delta)$. Итак, при $B=3$ справедлива оценка

$$
\left|I_{2}\right| \leqslant \max _{\Delta \leqslant \alpha \leqslant 1-\Delta} F(\alpha)\left(\frac{3}{2} \int_{0}^{1}\left|S_{1}^{2}(\alpha)\right| d \alpha+\frac{1}{2} \int_{0}^{1}\left|S_{2}^{2}(\alpha)\right| d \alpha\right) \ll \frac{U^{2}}{\log ^{4} N} .
$$

Преобразуем $S_{1}(\alpha)$ и $S_{2}(\alpha)$ при $|\alpha|<\Delta$, используя представление функции Чебышёва $\psi(x)$ в виде суммы по нулям дзета-функции Римана, мнимая часть которых ограничена $T=N^{0.4}$ (см. [4; c. 264, теорема 4.5]), и заменим в интеграле $I_{1}$ суммы $S_{1}(\alpha)$ и $S_{2}(\alpha)$ на $S_{3}(\alpha)$ и $S_{4}(\alpha)$ :

$$
\begin{gathered}
I_{1}=\frac{1}{\sqrt{2} \log ^{3} N} \int_{0}^{1} S_{3}^{2}(\alpha) S_{4}(\alpha) e^{-2 \pi i \alpha N} d \alpha+O\left(\frac{U^{2}}{\log ^{4} N}\right)+R, \\
S_{i}(\alpha)=\sum_{\frac{N}{3}-U<n<\frac{N}{3}+U} e^{2 \pi i \alpha n}-\sum_{|\rho|<T} \sum_{\frac{N}{3}-U<n<\frac{N}{3}+U} c_{i} n^{\rho-1} e^{2 \pi i \alpha n}, \quad c_{3}=n, c_{4}=\frac{n}{\sqrt{2}}
\end{gathered}
$$

(см. $[1 ;$ с. 39,42$])$.

Нам потребуется следующая оценка.

ЛЕмма 3. $\sum_{|\rho|<T} y^{\delta} \ll y e^{-c(\log y)^{0.2}} n p u T \leqslant y^{0.4}(\delta-$ действительная часть $\rho)$.

ДокАЗАТЕЛЬСТво см. [5; с. 210, лемма 11.20].

Из леммы следует, что $\max _{\Delta<\alpha<1-\Delta}\left|S_{4}(\alpha)\right| \ll U e^{-c \log ^{0.2} N}$, a $R \ll \frac{U^{2}}{\log ^{4} N}$.

И, наконец, $\int_{0}^{1} S_{3}^{2}(\alpha) S_{4}(\alpha) e^{-2 \pi i \alpha N} d \alpha=3 U^{2}\left(1+O\left(e^{-c \log ^{0.2} N}\right)\right)$.

Учитывая оценки $I_{1}$ и $I_{2}$, получаем асимптотическую формулу

$$
T(N, U)=\frac{3 U^{2}}{\sqrt{2} \log ^{3} N}+O\left(\frac{U^{2}}{\log ^{4} N}\right) .
$$

Доказательство теоремы завершено.

\section{СПИСОК ЛИТЕРАТУРЫ}

[1] Архипов Г.И., Буриев К., Чубариков В. Н. // Труды МИАН. 1997. Т. 218. C. 28-57. [2] Zhan Tao // Acta Math. Sinica (N.S.). 1991. V. 7.№ 3. P. 259-272. [3] Попов О. В. Арифметические приложения оценок сумм Г. Вейля от многочленов растущей степени // Дисс. ... канд. физ.-матем. наук. М.: МГУ, 1995. [4] Прахар К. Распределение простых чисел. М.: Mир, 1967. [5] Pan Chengdong, Pan Chengbiao. On the Goldbach Conjecture. Beijing: Science Press, 1981. 